\title{
\begin{tabular}{l|l} 
Mibraries & DSpace@MIT
\end{tabular}
}

\author{
MIT Open Access Articles
}

\section{First Principles Study of the Li-Bi-F Phase Diagram and Bismuth Fluoride Conversion Reactions with Lithium}

The MIT Faculty has made this article openly available. Please share how this access benefits you. Your story matters.

Citation: Doe, Robert E. et al. "First Principles Study of the Li--Bi--F Phase Diagram and Bismuth Fluoride Conversion Reactions with Lithium." Electrochemical and Solid-State Letters 12.7 (2009): A125-A128. (C) 2009 The Electrochemical Society

As Published: http://dx.doi.org/10.1149/1.3117249

Publisher: The Electrochemical Society

Persistent URL: http://hdl.handle.net/1721.1/52567

Version: Final published version: final published article, as it appeared in a journal, conference proceedings, or other formally published context

Terms of Use: Article is made available in accordance with the publisher's policy and may be subject to US copyright law. Please refer to the publisher's site for terms of use. 


\title{
First Principles Study of the Li-Bi-F Phase Diagram and Bismuth Fluoride Conversion Reactions with Lithium
}

\author{
Robert E. Doe, * Kristin A. Persson, Geoffroy Hautier, and Gerbrand Ceder*,z \\ Department of Materials Science and Engineering, Massachusetts Institute of Technology, Cambridge,
} Massachusetts 02139, USA

First principles calculations have been used to explore the $\mathrm{Li}-\mathrm{Bi}-\mathrm{F}$ ternary phase diagram. Our results confirm the thermodynamic stability of previously observed phases and find no new phases in this system. Electrochemical voltage profiles for the reaction of $\mathrm{Li}$ and $\mathrm{BiF}_{3}$ are in reasonable agreement with experiment. The driving force to form ternary $\mathrm{Li}-\mathrm{Bi}-\mathrm{F}$ intermediates is small. We also investigated the effect of particle size on the reaction voltage and find a potential decrease when nanoscale vs bulk Bi forms upon reacting $\mathrm{BiF}_{3}$ with $\mathrm{Li}$.

(C) 2009 The Electrochemical Society. [DOI: 10.1149/1.3117249] All rights reserved.

Manuscript submitted January 30, 2009; revised manuscript received March 9, 2009. Published April 13, 2009.

Alternatives to $\mathrm{Li}$-ion intercalation reactions have become the focus of considerable research efforts as the demand for higher energy density in Li batteries increases. ${ }^{1}$ One very promising alternative to intercalation is the conversion reaction in which $\mathrm{Li}^{+}$consumes the active electrode material, $\mathrm{MX}_{y}$ (M representing a metal cation and $\mathrm{X}$ representing the anion), reducing it to the metal, $\mathrm{M}^{0}$, and a corresponding lithium compound, $\mathrm{Li}_{z / y} \mathrm{X}$

$$
\mathrm{M}^{z+} \mathrm{X}_{y}+z \mathrm{Li}^{+} \underset{\text { charge }}{\stackrel{\text { discharge }}{\rightleftharpoons}} \mathrm{M}^{0}+y \mathrm{Li}_{z / y} \mathrm{X}
$$

In doing so, conversion reactions can take advantage of all energetically favorable valence states of the metal cation yielding specific capacities much higher than those of today's electrode materials. The reversibility of these reactions has only been demonstrated in recent years with the use of very small nanoparticles ( 1 to $20 \mathrm{~nm})$ in intimate contact with one another. ${ }^{2}$ This approach was initially used to establish reversible conversion with metal oxides, ${ }^{2}$ but promptly applied to reversible conversion of metal sulfides ${ }^{3}$ and metal nitrides ${ }^{4}$ thereafter. In general, these are considered anodic materials because the conversion reactions occur below $2 \mathrm{~V}$ vs $\mathrm{Li} / \mathrm{Li}^{+}$because of the more covalent nature of the metal-anion bonding. Consequently, only metal fluorides exhibit enough ionic character to yield high enough conversion reaction potentials to make them useful as cathodic materials. ${ }^{5}$ However, the inherently poor resistivity of fluoride materials requires an additional conductive matrix (e.g., carbon, $\left.\mathrm{MoO}_{3}\right)^{5}$ to ensure reversibility. Doing so has enabled nanocomposites based on several $3 \mathrm{~d}$ transition-metal fluorides (i.e., $\mathrm{Cr}, \mathrm{Fe}, \mathrm{Ni}$, and $\mathrm{Cu}$ ) to display significant capacities. ${ }^{5-8}$

$\mathrm{BiF}_{3}$ has been an attractive cathode material for several decades ${ }^{9}$ because of its high theoretical conversion potential $(3.21 \mathrm{~V}$ vs $\left.\mathrm{Li} / \mathrm{Li}^{+}\right)$and considerable gravimetric $(969 \mathrm{Wh} / \mathrm{kg})$ and volumetric ( $8042 \mathrm{Wh} / \mathrm{L})$ energy densities. However, only through the use of these nanocomposites has significant practical capacity ( $\approx 271 \mathrm{mAh} / \mathrm{g}$ of $\mathrm{BiF}_{3}$, corresponding to $90 \%$ theoretical capacity) been obtained. ${ }^{10,11}$ Utilizing a variety of characterization techniques, Bervas et al. indicated that $\mathrm{BiF}_{3}$ directly converts to a $\mathrm{LiF} / \mathrm{Bi}$ matrix via a two-phase reaction in which intermediate phases (with oxidation states between $\mathrm{Bi}^{3+}$ and $\mathrm{Bi}^{0}$ ) are not observed. They argued that pseudoplateaus observed in the voltage profiles of both the orthorhombic and tysonite $\mathrm{BiF}_{3}$ polymorphs are due to the varying electronic conductivity of the entire nanocomposite as metallic $\mathrm{Bi}$ is extruded (improving electronic conductivity) and consumed (making the nanocomposite more resistive). ${ }^{11}$ Regardless of the root cause for the pseudoplateaus, a significant voltage hysteresis is observed; an attribute shared among the variety of conversion reaction materials and generally attributed to the poor kinetics of electron conduction through the nanocomposite.

\footnotetext{
* Electrochemical Society Active Member

z E-mail: gceder@mit.edu
}

Recently we used first principles methods to investigate the conversion pathway of iron fluoride reacting with $\mathrm{Li}^{12}$ In doing so we speculated that iron fluoride conversion initially follows a thermodynamically equilibrated pathway, but that the reaction quickly moves to a nonequilibrium phase space due to the significant difference in diffusivity between $\mathrm{Li}$ and $\mathrm{Fe}$. Based on the hypothesis of a slowly diffusing $\mathrm{Fe}$, a different reaction path can be defined for charge and discharge, resulting in hysteresis. An additional finding of that study was that the formation of very small Fe particles (e.g., $1 \mathrm{~nm}$ ) causes the reaction voltage to significantly decrease from the bulk Fe value due to the substantial difference between the cohesive energy of bulk and nanosized Fe. In this article, we use first principles methods to study the equilibrium and nonequilibrium conversions of $\mathrm{BiF}_{3}$ and to also investigate the modification of the reaction voltage as a function of $\mathrm{Bi}$ particle size.

\section{Methodology}

The first principles results in this article are based on the spinpolarized generalized gradient approximation ${ }^{13}$ to the density functional theory formalism using pseudopotentials generated by the projector-augmented wave method ${ }^{14,15}$ and implemented with the Vienna Ab Initio Simulation Package. ${ }^{16}$ Pseudopotentials contained the valence states $1 s^{1} 2 s^{1} 2 p^{1}$ for $\mathrm{Li}, 5 \mathrm{~d}^{10} 6 \mathrm{~s}^{2} 6 \mathrm{p}^{3}$ for Bi (enabling lower lying d states to be treated as valence), and $2 \mathrm{~s}^{2} 2 \mathrm{p}^{5}$ for F. An energy cutoff of $550 \mathrm{eV}$ (higher than the default value of $400 \mathrm{eV}$ for fluorine) ensured planewave convergence, and Brillouin zone integration was performed on a $4 \times 4 \times 4$ grid for all $\mathrm{Li}-\mathrm{Bi}-\mathrm{F}$ compounds or a $12 \times 12 \times 12$ grid for $\mathrm{Li}$ and $\mathrm{Bi}$ metals. The total energy was minimized with the full relaxation of the atomic positions and cell parameters. For Bi nanoparticles only the $\Gamma$ point was used in reciprocal space. As it is expected that $\mathrm{Bi}$ forms as very small clusters, we computed the energy of a spherical $\mathrm{Bi}$ particle with $1 \mathrm{~nm}$ diameter to explore the cohesive energy change with size ${ }^{17}$ and its effect on the reaction potential of $\mathrm{BiF}_{3}$. The rhombohedral packing of bulk Bi (trigonal $R \overline{3} m$, SG no. 166) was employed because experimental evidence suggests that this symmetry is maintained for small Bi particles. ${ }^{10,11}$ The resulting cohesive energy was found to be $250 \mathrm{meV} / \mathrm{atom}$ lower than that calculated for bulk Bi.

The total energy of fluorine used to derive the $\mathrm{Li}-\mathrm{Bi}-\mathrm{F}$ phase diagram was determined from the experimental reaction enthalpy for the reaction

$$
2 \mathrm{Li}+\mathrm{F}_{2} \rightarrow 2 \mathrm{LiF}
$$

and the calculated energies for $\mathrm{Li}$ and $\mathrm{LiF}$. This enabled the total energy of fluorine to be determined by fitting the total energies of an insulator (i.e., $\mathrm{LiF}$ ) and a nontransition metal (i.e., Li) to the experimental reaction enthalpy, and minimized ab initio errors arising from the metal to anion charge transfer. ${ }^{18}$ The equivalent ab initio energy of $F_{2}$ was found to be $-2.784 \mathrm{eV} / \mathrm{f}$.u. based on this method. Similarly, the total energy of bismuth used to derive the $\mathrm{Li}-\mathrm{Bi}-\mathrm{F}$ 

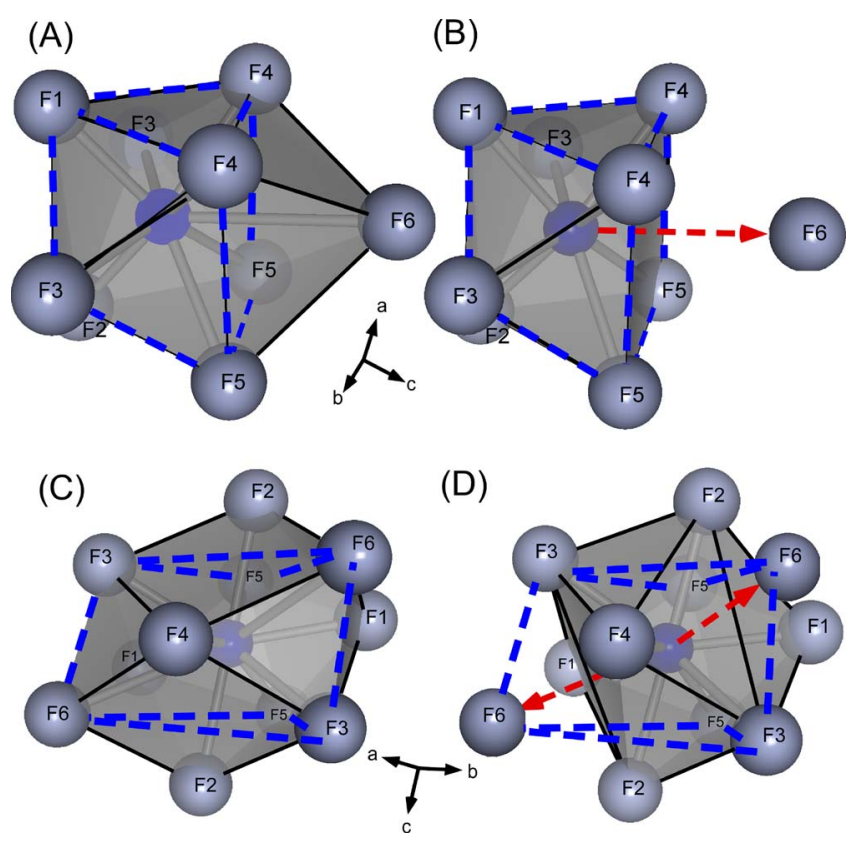

Figure 1. (Color online) The change in coordination from the (A) prototype orthorhombic $\mathrm{YF}_{3}$ to $(\mathrm{B}) o-\mathrm{BiF}_{3}$ and $(\mathrm{C})$ from the prototype tysonite $\mathrm{LaF}_{3}$ to (D) $t-\mathrm{BiF}_{3}$ is depicted. Both tysonite and the orthorhombic structure are based on metal-centered trigonal prisms (shown with dashed lines). One trigonal base is composed of atoms F1, F4, and F4, while the other is formed by atoms $\mathrm{F} 2, \mathrm{~F} 5$, and $\mathrm{F} 5$ in $o-\mathrm{BiF}_{3}$. Both trigonal bases are formed by atoms $\mathrm{F} 3, \mathrm{~F} 5$, and $\mathrm{F} 6$ in $t-\mathrm{BiF}_{3}$. The F6 fluorine atoms no longer bonding to $\mathrm{Bi}$ are depicted with dashed arrows.

phase diagram was determined from the experimental reaction enthalpy for the reaction

$$
3 \mathrm{Li}+\mathrm{BiF}_{3} \rightarrow \mathrm{Bi}+3 \mathrm{LiF}
$$

and the calculated energies for $\mathrm{Li}, \mathrm{BiF}_{3}$, and $\mathrm{LiF}$. This enabled the total energy of $\mathrm{Bi}$ to be determined by fitting the total energies of two insulators (i.e., $\mathrm{BiF}_{3}$ and $\mathrm{LiF}$ ) and a nontransition metal (i.e., $\mathrm{Li}$ ) to the experimental reaction enthalpy, thus minimizing ab initio errors arising from the significant difference between the electronic states in metallic and oxidized Bi.

\section{Structures Examined and the Predicted Li-Bi-F Ternary Phase Diagram}

The total energies of all binary and ternary Li-Bi-F phases listed in the Inorganic Crystal Structures Database (ICSD) have been calculated. ${ }^{19}$ The ICSD contains three polymorphs of $\mathrm{BiF}_{3}$ displaying space groups $P n m a$ (no. 62), $P \overline{4} 3 m$ (no. 215), and $F m \overline{3} m$ (no. 225). An additional calculation of $\mathrm{BiF}_{3}$ with the tysonite structure, from $\mathrm{LaF}_{3} P \overline{3} c 1$ (no. 165), was conducted as experimental efforts report the formation of this structure upon reconversion. ${ }^{10,11}$ Results of the total energy calculations confirmed that the orthorhombic Pnma structure, denoted $o-\mathrm{BiF}_{3}$, was lowest in energy, while the tysonite phase $\left(t-\mathrm{BiF}_{3}\right)$ was the next highest in energy $\left(132 \mathrm{meV} / \mathrm{f}\right.$.u. higher than $\left.o-\mathrm{BiF}_{3}\right)$. Both structures were based on a central $\mathrm{BiF}_{6}$ trigonal prism (outlined in dashed lines for $o-\mathrm{BiF}_{3}$ in Fig. $1 \mathrm{~A}$ and $\mathrm{B}$ and $t-\mathrm{BiF}_{3}$ in Fig. $1 \mathrm{C}$ and $\left.\mathrm{D}\right)$ to which additional fluorine coordinated through the three rectangular faces in each polymorph and through the two triangular faces in the tysonite structure. The coordination of $\mathrm{Bi}$ to $\mathrm{F}$ in $o-\mathrm{BiF}_{3}$ and $t-\mathrm{BiF}_{3}$ was reduced from that of their respective prototypes, $\mathrm{YF}_{3}$ and $\mathrm{LaF}_{3}$, due to the stereochemically active lone pair on Bi. Figure 1 contrasts the ninefold coordination of the metal in $\mathrm{YF}_{3}$ (Fig. 1A), which exhibits metal-fluorine bonds in the range $2.281-2.538 \AA{ }^{20},{ }^{20}$ with the reduced eightfold coordination of $\mathrm{Bi}$ in $o-\mathrm{BiF}_{3}$. The calculated $\mathrm{M}-\mathrm{F}$

\begin{tabular}{|c|c|c|c|c|c|}
\hline \multicolumn{6}{|c|}{$\begin{array}{l}\text { Table I. The bismuth-fluorine bond lengths from the opt } \\
\text { structures for both } o-\mathrm{BiF}_{3} \text { and } t-\mathrm{BiF}_{3} \text {. The "Bi-F6" bond do } \\
\text { genuinely coordinate in } \mathrm{BiF}_{3} \text {, but would coordinate for bot } \\
\text { respective prototypes. } \\
\text { Calculated bismuth distance to nearest neighbors }\end{array}$} \\
\hline \multicolumn{3}{|c|}{$o-\mathrm{BiF}_{3}$} & \multicolumn{3}{|c|}{$t-\mathrm{BiF}_{3}$} \\
\hline Bond & F site & $\begin{array}{c}\overline{\text { Length }} \\
(\AA)\end{array}$ & Bond & F site & $\begin{array}{c}\text { Length } \\
(\AA)\end{array}$ \\
\hline Bi-F1 $(\times 1)$ & $4 \mathrm{c}$ & 2.265 & Bi-F1 $(\times 2)$ & $4 \mathrm{c}$ & 2.388 \\
\hline Bi-F2 $(\times 1)$ & $4 c$ & 2.288 & $\mathrm{Bi}-\mathrm{F} 2(\times 2)$ & $4 \mathrm{c}$ & 2.421 \\
\hline $\mathrm{Bi}-\mathrm{F} 3(\times 2)$ & $8 \mathrm{~d}$ & 2.385 & Bi-F3 $(\times 2)$ & $8 d$ & 2.450 \\
\hline $\mathrm{Bi}-\mathrm{F} 4(\times 2)$ & $8 \mathrm{~d}$ & 2.437 & $\mathrm{Bi}-\mathrm{F} 4(\times 1)$ & $8 d$ & 2.469 \\
\hline $\mathrm{Bi}-\mathrm{F} 5(\times 2)$ & $8 d$ & 2.520 & $\mathrm{Bi}-\mathrm{F} 5(\times 2)$ & $8 \mathrm{~d}$ & 2.586 \\
\hline Bi-F6 $(\times 1)$ & $4 \mathrm{c}$ & 3.340 & Bi-F6 $(\times 2)$ & $4 \mathrm{c}$ & 3.080 \\
\hline
\end{tabular}

bond lengths listed in Table I show that the eight nearest-neighbor fluorine reside $2.265-2.520 \AA$ from $\mathrm{Bi}$, while the ninth fluorine is nearly $1 \AA$ further. This is consistent with earlier investigations concluding that $o-\mathrm{BiF}_{3}$ does not contain $\mathrm{Bi}$ in true ninefold coordination. ${ }^{20}$ We observed a similar behavior for the $\mathrm{Bi}$ in $t-\mathrm{BiF}_{3}$. Figure 1 depicts the 11-fold coordination of lanthanum in the metalcentered polyhedron of $\mathrm{LaF}_{3}{ }^{21}$ in contrast with the reduced ninefold coordination of $\mathrm{Bi}$ in $t-\mathrm{BiF}_{3}$. The calculated $\mathrm{Bi}-\mathrm{F}$ bond lengths, displayed in Table I, show that nine fluorine atoms are situated 2.388-2.586 $\AA$ from Bi and that the two next-nearest fluorine atoms are $3.080 \AA$ from Bi.

The cubic $\mathrm{BiF}_{3}$ polymorphs, $P \overline{4} 3 m$ and $F m \overline{3} m$, are essentially structurally and energetically equivalent to one another, but have a substantially higher energy than $o-\mathrm{BiF}_{3}(1.06 \mathrm{eV} /$ f.u. higher). Total energies were also calculated for the $\mathrm{BiF}_{5}$ composition and the binary $\mathrm{Li}-\mathrm{Bi}$ alloys known for this system. We also made extensive effort to search for ternary $\mathrm{Li}-\mathrm{Bi}-\mathrm{F}$ phases beyond the previously identified $\mathrm{LiBiF}_{4}$ and $\mathrm{LiBiF}_{6}$. Some potential structures were obtained by topotactic $\mathrm{Li}^{+}$insertion in $o-\mathrm{BiF}_{3}$ (both octahedral and tetrahedral lithium coordinations in $\mathrm{Li}_{0.25} \mathrm{BiF}_{3}$ ), through $\mathrm{Li} / \mathrm{Bi}$ exchange in $\mathrm{LiBiF}_{4}$, and spinel compositions containing $\mathrm{Bi}^{3+}$. We applied a structure prediction algorithm to this system, which has been successfully applied to the prediction of binary structures ${ }^{22}$ and is now being extended to ternary structures. This structure predictor used a probabilistic structure correlation scheme to suggest possible candidate structures by correlation with other known systems. The search encompassed ternary structures in which the Bi oxidation state was between +3 and +5 , yielding 122 probable $\mathrm{Li}-\mathrm{Bi}-\mathrm{F}$ phases at 16 compositions. The total energy was calculated for the top 53 of the 122 candidate structures although ultimately none of these were found to be stable in the $\mathrm{Li}-\mathrm{Bi}-\mathrm{F}$ ternary phase diagram.

The ternary $\mathrm{Li}-\mathrm{Bi}-\mathrm{F}$ ground-state phase diagram derived from these energy calculations is depicted in Fig. 2. The phase space of a ternary phase diagram at $0 \mathrm{~K}$ is divided into triangles, which indicate the coexistence of three phases. Compositions that are thermodynamically stable are denoted with a filled black circle, signifying that they exhibit an energy lower than any linear combination of other compounds that sum up to the same composition (and consequently they become part of the convex lowest energy hull). Compositions denoted by a cross are thermodynamically unstable as they exhibit an energy higher than that of the mixture of the stable phases composing that triangle. It is possible that a metastable formation of such compounds does take place if a stable phase cannot form due to slow-component diffusion or if nucleation is limited for certain compounds. Figure 2 also shows the equilibrium voltages with respect to lithium metal within each three-phase region.

Because the edge- and corner-sharing $\mathrm{Bi}-\mathrm{F}$ polyhedra of $o-\mathrm{BiF}_{3}$ were arranged in a manner that could reasonably accommodate either octahedral or tetrahedral coordination of $\mathrm{Li}^{+}$, the topotactic insertion of $o-\mathrm{BiF}_{3}$ was also explored even though intermediate oxi- 


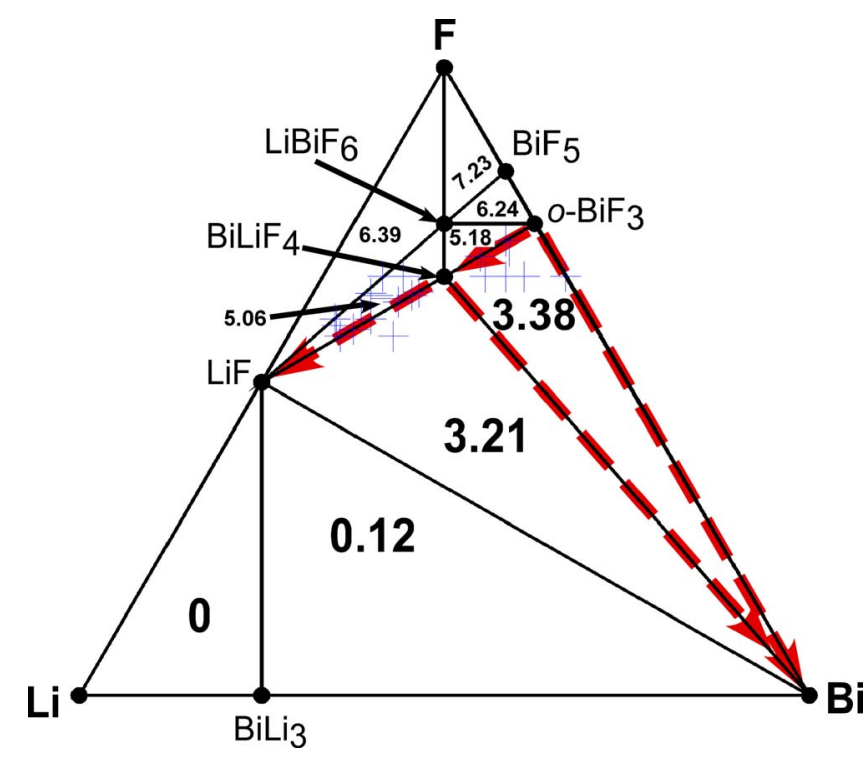

Figure 2. (Color online) The Li-Bi-F ternary phase diagram derived from first principles calculations. Thermodynamically stable phases are labeled and shown with a closed black circle, while unstable phases are represented with a cross. The equilibrium voltage of lithiation, calculated from the lithium chemical potential, is also denoted within each three-phase region. The dashed arrows represent the equilibrium decomposition path for the lithiation of $\mathrm{BiF}_{3}$.

dation states between +3 and 0 are very rare for $\mathrm{Bi}$ in the solid state. In the dilute limit, $x=1 / 4$ in $\mathrm{Li}_{x} \mathrm{BiF}_{3}$, octahedral $\mathrm{Li}^{+}$coordination was favored over tetrahedral coordination by $13.8 \mathrm{meV} / \mathrm{atom}$, however neither inserted structure was found to be thermodynamically stable (octahedrally coordinated $\mathrm{Li}_{0.25} \mathrm{BiF}_{3}$ was $117 \mathrm{meV} /$ atom too high). This supports experimental observation, which indicates that both polymorphs convert directly to metallic $\mathrm{Bi}$ and $\mathrm{LiF}$ in a twophase, three-electron reduction that does not require any topotactic $\mathrm{Li}^{+}$insertion. ${ }^{11}$ Indeed, Fig. 2 indicates that no ternary phases are predicted to be thermodynamically stable other than the two previously known phases, $\mathrm{LiBiF}_{6}$ and $\mathrm{LiBiF}_{4}$; other ternary compositions being a minimum $\sim 50 \mathrm{meV} /$ atom from the convex hull.

\section{The Calculated Equilibrium Voltage Profile}

The calculated voltage profiles ${ }^{23}$ for the reaction of $o-\mathrm{BiF}_{3}$ with $\mathrm{Li}$ under thermodynamic equilibrium are presented in Fig. 3 for the formation of both bulk (squares) and nanoscale (circles) Bi. The initial conversion of $\mathrm{BiF}_{3}$ to $\mathrm{LiBiF}_{4}$ and $\mathrm{Bi}$ occurred at $3.38 \mathrm{~V}$ for bulk $\mathrm{Bi}$ formation and at $3.29 \mathrm{~V}$ when nanoscale $\mathrm{Bi}$ formed. After this conversion was completed, the voltage stepped down to 3.21 and $3.13 \mathrm{~V}$ for the formation of $\mathrm{LiF}$ and bulk or nanoscale $\mathrm{Bi}$, respectively. $o-\mathrm{BiF}_{3}$ and $t-\mathrm{BiF}_{3}$ were close enough in energy to yield the same convex hulls and phase diagram for $T=0 \mathrm{~K}$. As a result the calculated conversion of $t-\mathrm{BiF}_{3}$ (not shown here) also occurred with a single voltage step of $340 \mathrm{mV}$ from $3.55 \mathrm{~V}$ as $\mathrm{LiBiF}_{4}$ formed to $3.21 \mathrm{~V}$ as $\mathrm{LiF}$ formed. The step was larger due to the higher energy of $t-\mathrm{BiF}_{3}$. When nanoscale $\mathrm{Bi}$ formed instead of bulk $\mathrm{Bi}$, the two plateau voltages were 3.47 and $3.13 \mathrm{~V}$. For comparison with the theoretical voltage profiles, Fig. 3 also shows the potential profile obtained during the galvanostatic intermittent titration technique (GITT) experiments.

\section{Discussion}

Even with exhaustive searching we found no new ternary compounds in the $\mathrm{Li}-\mathrm{Bi}-\mathrm{F}$ system, indicating that its experimental coverage may be rather complete. Unlike what was found in our study of the $\mathrm{Li}-\mathrm{Fe}-\mathrm{F}$ system, ${ }^{12}$ we found that the reaction path of $\mathrm{BiF}_{3}$ with $\mathrm{Li}$, shown in Fig. 2, was rather straightforward. This path

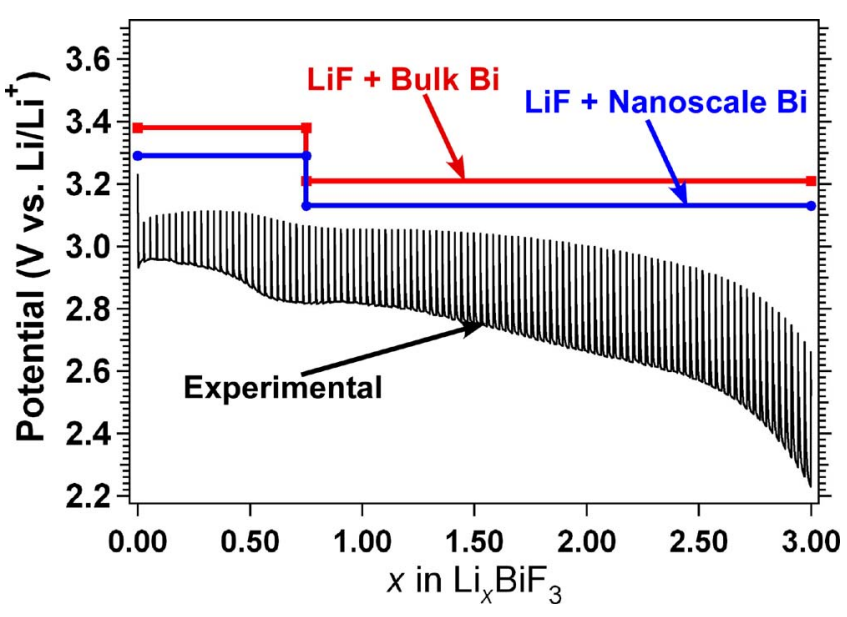

Figure 3. (Color online) The calculated voltage profiles for $o-\mathrm{BiF}_{3}$ conversion to $\mathrm{LiF}$ and bulk Bi (squares) and nanoscale $\mathrm{Bi}$ (circles). For comparison, the experimental GITT profile for discharge of an $o-\mathrm{BiF}_{3} / \mathrm{MoS}_{2}$ nanocomposite (black) is also plotted above. It was provided to us by Professor Glenn Amatucci of Rutgers, The State University of New Jersey.

shows that, under equilibrium conditions, $\mathrm{BiF}_{3}$ conversion takes place via two disproportionation steps: initially $\mathrm{BiF}_{3}$ disproportionates into metallic $\mathrm{Bi}$ and $\mathrm{LiBiF}_{4}$, which subsequently decomposes into $\mathrm{LiF}$ and additional $\mathrm{Bi}$. The phase diagram also indicates that the continued lithiation associated with the formation of $\mathrm{Li}_{3} \mathrm{Bi}$ is possible at voltages $<1 \mathrm{~V}$ vs $\mathrm{Li} / \mathrm{Li}^{+}$. Indeed such an observation is noted in early work regarding the electrochemical activity of $\mathrm{BiF}_{3} .{ }^{9}$

Whether $\mathrm{LiBiF}_{4}$ forms as an intermediate when $\mathrm{BiF}_{3}$ is lithiated is unclear. A significant topological relationship between $\mathrm{BiF}_{3}$ and $\mathrm{LiBiF}_{4}$ is not evident, and previous electrochemical investigations indicate that no reaction intermediates form. ${ }^{10,11}$ There are multiple reasons why $\mathrm{LiBiF}_{4}$ may not form. For instance, the Li voltages calculated and shown in our phase diagram indicate that the driving force to form $\mathrm{LiBiF}_{4}$ is small. It forms at $3.29 \mathrm{~V}$ from $\mathrm{BiF}_{3}$, but is only stable down to $3.13 \mathrm{~V}$ before decomposing to $\mathrm{Bi}$ and $\mathrm{LiF}$. Hence, any polarization reducing the voltage may lead to direct $\mathrm{Bi}$ and $\mathrm{LiF}$ formation, even in the very early part of discharge. Kinetically $\mathrm{Bi}$ and $\mathrm{LiF}$ formation is probably much easier than $\mathrm{LiBiF}_{4}$ formation. Our calculated energies indicate that if $\mathrm{LiBiF}_{4}$ were not to form, $\mathrm{BiF}_{3}$ would decompose to $\mathrm{LiF}$ and nanoscale $\mathrm{Bi}$ at $3.17 \mathrm{~V}$. Such a lower and flatter voltage is in better agreement with the experimental data shown in Fig. 3.

Given the large amount of polarization in the experimentally measured voltage curve, ${ }^{10,11}$ it is not straightforward to compare with our calculated curves in Fig. 3, although it does seem that the predicted voltage profile of $\mathrm{BiF}_{3}$ is about $\sim 0.3 \mathrm{~V}$ higher in magnitude than the "equilibrated" voltage curve obtained during the current relaxation portion of the GITT experiment. The difference between the calculated and the experimental equilibrated voltage decreases to $\sim 0.2 \mathrm{~V}$ when accounting for the precipitation of very small particles by using the energy of a $1 \mathrm{~nm}$ Bi particle, a voltage decrease of $85 \mathrm{mV}$ from that expected using the bulk energy as illustrated in Fig. 3 for $o-\mathrm{BiF}_{3}$. The decrease in cohesive energy from the bulk metal to a $1 \mathrm{~nm}$ particle is significantly less for $\mathrm{Bi}$ than the change we observed with Fe. Bulk Bi displays only about one-half of the cohesive energy of bulk Fe to begin with. ${ }^{12}$ Therefore we speculate that bulk metals with a small cohesive energy may cause a less dramatic voltage drop when precipitating as nanoscale particles during a conversion reaction. While the voltage curves that are experimentally obtained under applied current show some steplike features, the voltage obtained after relaxing the system is almost independent of the state of charge, indicating that it may be the voltage of unreacted $\mathrm{BiF}_{3}$ in metastable equilibrium with $\mathrm{LiF}$ and Bi. 
In a previous paper on conversion in the $\mathrm{Li}-\mathrm{Fe}-\mathrm{F}$ system, we advanced a theory for estimating the nonequilibrium reaction paths and voltages. The hypothesis was that metal diffusion (in or out of the host) is rate limiting. Applying the same ideas to this system would imply that upon discharge $\mathrm{Bi}$ is reduced to the lowest possible valence state in the fluoride host before it precipitates out. Upon reconversion ( $\mathrm{Li}$ extraction) maximal oxidation of $\mathrm{Bi}$ in the host would occur. In the Li-Bi-F system these assumptions do not lead to a path that is very different from the equilibrium path. Given that $\mathrm{Bi}^{3+}$ is the lowest oxidation state that is observed in oxidized materials, this kinetic model would predict a direct conversion of $\mathrm{BiF}_{3}$ to $\mathrm{Bi}$ and LiF, which is indeed what is observed.

When considering reconversion from $\mathrm{Bi}$ and $\mathrm{LiF}$, we can predict an analogous nonequilibrium effect on the reaction voltage because the reaction will attempt to form a structure with maximally oxidized $\mathrm{Bi}$ in a rapid manner. Reconversion to $\mathrm{BiF}_{5}$ is only possible above 5-6 V, indicating that it is unlikely. Reconversion under nonequilibrium conditions from nanoscale $\mathrm{Bi}$ and $\mathrm{LiF}$ initiates from $3.13 \mathrm{~V}$ and quickly increases to 3.29 or $3.47 \mathrm{~V}$ if reconversion forms $o-\mathrm{BiF}_{3}$ or $t-\mathrm{BiF}_{3}$, respectively. Although the magnitude of the predicted voltage under nonequilibrium reconversion is significantly different from that observed, the size of the voltage step is still in reasonable agreement with that of experiment, ${ }^{10,11}$ particularly when taking into account the experimental observation that reconversion always forms $t-\mathrm{BiF}_{3}$.

\section{Conclusions}

The Li-Bi-F ternary phase diagram derived from first principles calculations confirms the stability of all previously identified ternary phases and does not identify any new phases. The voltage profile for the conversion reaction of $\mathrm{BiF}_{3}$ and $\mathrm{Li}$ to $\mathrm{LiF}$ and $\mathrm{Bi}$, calculated from the ternary phase diagram, is in reasonable agreement with experiment. We report that the precipitation of nanoscale Bi lowers the conversion voltage by $85 \mathrm{mV}$ due to loss of the cohesive energy when $1 \mathrm{~nm}$ Bi particles form. We find that the equilibrium and nonequilibrium reaction paths do not differ greatly, and $\mathrm{Bi}$ does not access any valence states below +3 in fluorides. The formation of the reaction intermediate, $\mathrm{LiBiF}_{4}$, is unlikely during both discharge and charge because the driving force is very small.

\section{Acknowledgments}

This research was supported by the Intelligence Community Postdoctoral Research Fellowship Program award no. HM1582-061-2012. This research was supported in part by the National Science Foundation through TeraGrid resources provided by the Pittsburgh Supercomputing Center. The authors thank Professor Glenn Amatucci for helpful discussion and for providing the experimental data for comparison with the authors' first principles results.

Massachusetts Institute of Technology assisted in meeting the publication costs of this article.

\section{References}

1. M. Armand and J.-M. Tarascon, Nature (London), 451, 652 (2008).

2. P. Poizot, S. Laruelle, S. Grugeon, L. Dupont, and J. M. Tarascon, Nature (London), 407, 496 (2000).

3. P. Poizot, S. Laruelle, S. Grugeon, and J. M. Tarascon, J. Electrochem. Soc., 149, A1212 (2002).

4. N. Pereira, L. C. Klein, and G. G. Amatucci, J. Electrochem. Soc., 148, A262 (2002).

5. G. G. Amatucci and N. Pereira, J. Fluorine Chem., 128, 243 (2007).

6. F. Badway, F. Cosandey, N. Pereira, and G. G. Amatucci, J. Electrochem. Soc., 150, A1318 (2003)

7. F. Badway, A. N. Mansour, N. Pereira, J. F. Al-Sharab, F. Cosandey, I. Plitz, and G G. Amatucci, Chem. Mater, 19, 4129 (2007)

8. F. Badway, N. Pereira, F. Cosandey, and G. G. Amatucci, J. Electrochem. Soc. 150, A1209 (2003).

9. P. Fiordiponti, S. Panero, G. Pistoia, and C. Temperoni, J. Electrochem. Soc., 125, 511 (1978)

10. M. Bervas, F. Badway, L. C. Klein, and G. G. Amatucci, Electrochem. Solid-State Lett., 8, A179 (2005)

11. M. Bervas, A. N. Masour, W.-S. Yoon, J. F. Al-Sharab, F. Badway, F. Cosandey, L. C. Klein, and G. G. Amatucci, J. Electrochem. Soc., 153, A799 (2006).

12. R. E. Doe, K. A. Persson, Y. S. Meng, and G. Ceder, Chem. Mater., 20, 5274 (2008).

13. J. P. Perdew, K. Burke, and M. Ernzerhof, Phys. Rev. Lett., 77, 3865 (1996).

14. P. E. Blochl, Phys. Rev. B, 50, 17953 (1994).

15. G. Kresse and J. Joubert, Phys. Rev. B, 59, 1758 (1999).

16. G. Kresse and J. Furthmuller, Phys. Rev. B, 54, 169 (1996)

17. L. F. Cao, G. Y. Xu, D. Xie, M. X. Guo, L. Luo, Z. Li, and M. P. Wang, Phys Status Solidi B, 243, 2745 (2006)

18. L. Wang, T. Maxisch, and G. Ceder, Phys. Rev. B, 73, 195107 (2006).

19. Fachinformationszentrum (FIZ) Karlsruhe, Inorganic Crystal Structure Database, http://icsdweb.fiz-karlsruhe.de/

20. A. K. Cheetham and N. Norman, Acta Chem. Scand., Ser. A, 28, 55 (1974).

21. A. Abaouz, A. Taoudi, and J. P. Laval, J. Solid State Chem., 130, 277 (1997).

22. C. C. Fischer, K. Tibbetts, D. Morgan, and G. Ceder, Nature Mater, 5, 641 (2006).

23. M. K. Aydinol, A. F. Kohan, G. Ceder, K. Cho, and J. Joannopoulos, Phys. Rev. B, 56, 1354 (1997). 